\title{
DENSITY OF A NORMAL SUBGROUP OF THE INVERTIBLES IN CERTAIN MULTIPLIER ALGEBRAS
}

\author{
P. W. NG AND TRACY ROBIN
}

Abstract. Let $\mathscr{A}$ be a unital separable simple $\mathrm{C}^{*}$-algebra. Let $G L(\mathscr{M}(\mathscr{A} \otimes \mathscr{K}))$ be the group of invertible elements of the multiplier algebra of the stabilization of $\mathscr{A}$, and let $N \subseteq G L(\mathscr{M}(\mathscr{A} \otimes$ $\mathscr{K}))$ be any (algebraic) normal subgroup that properly contains the scalar invertibles.

Then

$$
\bar{N}^{\text {strict }}=\mathscr{M}(\mathscr{A} \otimes \mathscr{K}),
$$

where $\bar{N}^{\text {trict }}$ is the closure of $N$ in the strict topology.

Mathematics subject classification (2010): 46L05.

Keywords and phrases: $\mathrm{C}^{*}$-algebras, multiplier algebras, general linear group, normal subgroup, Busby invariant, absorbing extensions.

\section{REFERENCES}

[1] W. Arveson, Notes on extensions of $C^{*}$-algebras, Duke Mathematical Journal, 44 (1977), no. 2, 329-355.

[2] B. BlackADAR, $K$-theory for operator algebras, second edition, Mathematical Sciences Research Institute Publications. Cambridge University Press, Cambridge, 1998.

[3] L. G. Brown, R. G. Douglas and P. A. Fillmore, Unitary equivalence modulo the compact operators and extensions of $C^{*}$-algebras, Proceedings of a Conference on Operator Theory (Dalhousie Univ., Halifax, N. S., 1973), 58-128. Lecture Notes in Math., 345, Springer, Berlin, 1973.

[4] K. Davidson, $C^{*}$-algebras by example, Fields Institute Monographs, 6, American Mathematical Society, Providence, RI, 1996.

[5] G. A. Elliott And D. Kucerovsky, An abstract Voiculescu-Brown-Douglas-Fillmore absorption theorem, Pacific Journal of Mathematics 198 (2001), no. 2, 385-409.

[6] G. A. ElliotT AND M. RoRdam, The automorphism group of the irrational rotation $C^{*}$-algebra, Comm. Math. Phys. 155 (1993), no. 1, 3-26.

[7] C. K. Fong, C. R. Miers AND A. R. Sourour, Lie and Jordan ideals of operators on Hilbert space, Proc. Amer. Math. Soc. 84 (1982), no. 4, 516-520.

[8] B. Fuglede, A Commutativity Theorem for Normal Operators, Proceedings of the National Academy of Sciences of the United States of America, 1/15/1950, vol. 36, issue 1, pp. 35-40.

[9] B. Fuglede And R. Kadison, Determinant theory in finite factors, Ann. of Math. 55 (1952), 520 530.

[10] W. Fulton And J. Harris, Representation theory. A first course, Graduate Texts in Mathematics, 129 (1991) Readings in Mathematics, Springer-Verlag, New York.

[11] D. A. HERRERO, Approximation of Hilbert space operators, vol. 1, second edition, Pitman Research Notes in Mathematics Series, 224, Longman Scientific and Technical, Harlow; copublished in the United States with John Wiley and Sons, Inc., New York, 1989.

[12] I. N. HeRsteIn, On the Lie and Jordan rings of a simple associative ring, Amer. J. Math. 77 (1955), 270-285.

[13] I. N. HeRstein, Topics in Ring Theory, University of Chicago Press 1969.

[14] A. Hopenwasser And V. PAUlsen, Lie ideals in operator algebras, J. Operator Theory 52 (2004), no. $2,325-340$. 
[15] T. D. Hudson, L. Marcoux And A. R. Sourour, Lie ideals in triangular operator algebras, Trans. Amer. Math. Soc. 350 (1998), no. 8, 3321-3339.

[16] K. K. Jensen And K. Thomsen, Elements of KK-theory, Mathematics: Theory and Applications, Birkhaeuser Boston, Inc., Boston, MA 1991.

[17] N. KUIPER, The homotopy type of the unitary group of Hilbert space, Topology 3 (1965), 19-30.

[18] R. V. KADISON, Infinite unitary groups, Trans. Amer. Math. Soc. 72 (1952), 386-399.

[19] R. V. KADISON, Infinite general linear groups, Trans. Amer. Math. Soc. 76, (1954), 66-91.

[20] R. V. KADISON, On the general linear group of infinite factors, Duke Math. J. 22 (1955), 119-122.

[21] G. G. KaSParov, Topological invariants of elliptic operators, I: K-homology, Izv. Adad. Nauk SSSR SEr. Math. 39 (1975), 796-838, in Russian, translated in Math. USSR Izv. 9 (1975), no. 4, 751-794.

[22] G. G. KASPAROV, The operator K-functor and extensions of $C^{*}$-algebras, Izv. Akad. Nauk. SSSR Ser. Math. 44 (1980), no. 3, 571-636, 719, in Russian; translation in Math. USSR Izvestija 16 (1981), 513-572.

[23] D. KUCEROVSKY AND P. W. NG, The corona factorization property and approximate unitary equivalence, Houston J. Math. 32 (2006), no. 2, 531-550.

[24] H. LiN, Generalized Weyl-von Neumann theorems (II), Math. Scand. 77 (1995), 129-147.

[25] H. Lin, Extensions by $C^{*}$-algebras with real rank zero II, Proc. London Math. Soc. 71 (1995), 641674.

[26] H. LiN, An introduction to the classification of amenable $C^{*}$-algebras, World Scientific Publishing Co., Inc., River Edge, NJ, 2001.

[27] H. Lin, Stable unitary equivalence of homomorphisms, J. Operator Theory 47 (2002), no. 2, 343-378.

[28] L. W. Marcoux, On the linear span of the projections in certain simple $C^{*}$-algebras, Indiana Univ. Math. J. 51 (2002), no. 3, 753-771.

[29] L. W. Marcoux, Projections, commutators and Lie ideals in $C^{*}$-algebras, Math. Proc. R. Ir. Acad. 110A (2010), no. 1, 31-55.

[30] L. W. Marcoux AND G. J. Murphy, Unitarily-invariant linear spaces in $C^{*}$-algebras, Proc. Amer. Math. Soc. 126 (1998), no. 12, 3597-3605.

[31] L. W. Marcoux AND A. R. Sourour, Conjugation-invariant subspaces and Lie ideals in nonselfadjoint operator algebras, J. London Math. Soc. (2) 65 (2002), no. 2, 493-512.

[32] J. A. MingO, K-theory and multipliers of stable $C^{*}$-algebras, Trans. Amer. Math. Soc. 299 (1987), no. $1,397-411$.

[33] P. W. NG AND E. RuIZ, The automorphism group of a simple tracially AI algebra, Comm. Math. Phys. 280 (2008), no. 2, 427-444.

[34] P. W. NG AND E. RUIZ, The structure of the unitary groups of certain simple $C^{*}$-algebras, Houston J. Math. 35 (2009), no. 4, 1203-1232.

[35] P. W. NG AND E. RuIZ, The automorphism group of a simple $\mathscr{Z}$-stable $C^{*}$-algebra, Trans. Amer. Math. Soc. 365 (2013), no. 8, 4081-4120.

[36] P. W. NG AND E. RUIZ, Simplicity of the projective unitary group of the multiplier algebra of a simple stable nuclear $C^{*}$-algebra, Rocky Mountain J. Math. 40 (2010), no. 5, 1649-1665.

[37] P. W. NG AND E. RUIZ, On the structure of the projective unitary group of the multiplier algebra of a stable nuclear $C^{*}$-algebra, J. Operator Theory $\mathbf{6 8}$ (2012), no. 2, 549-565.

[38] L. ROBERT, On the Lie ideals of $C^{*}$-algebras, preprint, a copy is available at http://arxiv.org/abs/1505.04503.

[39] M. TAKESAKI, Theory of operator algebras, I, Springer-Verlag (1979), New York-Heidelberg.

[40] N. E. WegGe-Olsen, $K$-theory and $C^{*}$-algebras. A friendly approach, Oxford University Press, New York, 1993.

[41] S. Zhang, Trivial $K_{1}$-flow of AF algebras and finite von Neumann algebras, Journal of Functional Analysis 92 (1990), 77-91. 